\title{
Primary mucinous adenocarcinoma of the lung: A case report and review of the literature
}

\author{
YAN LIU $^{1,2}$, HE-LONG ZHANG ${ }^{3}$, JIA-ZHUAN MEI ${ }^{1}$, YAN-WEI GUO ${ }^{1}$, \\ RUI-JUN LI ${ }^{1}$, SI-DONG WEI ${ }^{2}$, FU TIAN ${ }^{2}$, LU YANG ${ }^{2}$ and HUI WANG ${ }^{2}$ \\ ${ }^{1}$ Department of Oncology; ${ }^{2}$ The Academician Workstation, Zhengzhou People's Hospital, Zhengzhou, Henan 450003; \\ ${ }^{3}$ Department of Oncology, Tangdu Hospital, Fourth Military Medical University, Xi'an, Shaanxi 710038, P.R. China
}

Received November 12, 2015; Accepted April 13, 2017

DOI: $10.3892 / \mathrm{ol} .2017 .6615$

\begin{abstract}
Mucinous adenocarcinoma is an unusual histological type of lung cancer, and its clinicopathological feature is distinctive from that of other histopathological types of lung adenocarcinoma. Mucinous adenocarcinoma has a mucus-producing function, which explains its name. The present study reports a rare case of a mucus-producing adenocarcinoma of the lung. A 60-year-old Chinese female patient was diagnosed with mucinous adenocarcinoma of the lung, which manifested as respiratory symptoms, including fever, cough and expectoration. The patient received thoracic exploratory operation and right pneumonectomy, since the above respiratory symptoms seriously affected her daily life, and other inspections could not establish the diagnosis. Histopathology revealed no mutations in epidermal growth factor receptor. The patient received adjuvant chemotherapy using taxol and cisplatin. However, metastases in the left lung were detected 7 months after the operation. Pemetrexed and cisplatin were used as the second-line treatment. The patient survived 3 years after the initial diagnosis. The present study reports a rare mucus-producing adenocarcinoma of the lung, which is of bad prognosis. Therefore, further studies on this type of cancer are urgently required.
\end{abstract}

\section{Introduction}

Lung cancer is the leading cancer in terms of mortality and morbidity in the world, and adenocarcinomas are common histological types of lung cancer $(1,2)$. The International Society for the Study of Lung Cancer (IASLC), the American Thoracic Society and the European Respiratory Society jointly published a histological classification of lung adenocarcinomas in 2011 (3). The main interest of such classification is its

Correspondence to: Dr Yan Liu, Department of Oncology, Zhengzhou People's Hospital, 33 Huanghe Road, Zhengzhou, Henan 450003, P.R. China

E-mail: liuyan9917@163.com

Key words: lung mucinous adenocarcinoma, CT scan prognostic value, since histological type is closely associated with clinical, pathological and molecular parameters $(4,5)$.

Mucinous adenocarcinoma is the rarest type of lung adenocarcinoma (6). According to a recent classification (3), mucinous adenocarcinoma includes mucinous adenocarcinoma in situ, minimally invasive adenocarcinoma and invasive mucinous adenocarcinoma. These tumors tend to present KRAS mutations and commonly lack thyroid transcription factor-1 expression, and computed tomography (CT) usually indicates nodules of consolidation with air bronchograms that are generally multinodular and multilobular in distribution (5). Mucinous adenocarcinoma of the lung is morphologically characterized by tall columnar cells with abundant cytoplasm that contain varying amounts of mucin $(6,7)$. Mucus secreted by cancer cells can commonly be discharged as sputum. However, if airway obstruction happens, obstructive pneumonia occurs immediately, since the mucus fails to drain (8-10).

\section{Case report}

The patient was a 60-year-old female. On October 2011, the patient developed fever, with a temperature fluctuation between 37.5 and $39.0^{\circ} \mathrm{C}$, in addition to cough and yellow sputum, but no chills or shivering, abdominal pain or diarrhea, sore throat, urinary frequency or urgency, or dysuria. In the Hospital of Traditional Chinese Medicine, a CT scan of the chest revealed a right lung space-occupying lesion, and the effect of anti-infective therapy was poor. On April 16, 2012, when the patient was referred to Zhengzhou People's Hospital (Zhengzhou, China), cough seriously affected her daily life. Fiberoptic bronchoscope examination revealed a mucinous membrane with black spots in the right upper lobe anterior segmental bronchus. Bronchial brush cytology revealed well differentiated respiratory epithelial cells and sporadic neutrophilic granulocytes without malignant cells. Sputum examination revealed gram-negative bacillus and gram-positive cocci, no fungus spores or hypha, and no malignant cells. Sputum culture identified Escherichia coli but no fungus. Thus, anti-infective therapy (cefoperazone combined with levofloxacin) was administered for 2 weeks, but its effect was poor. Following treatment, chest CT scans (Fig. 1A) revealed that the right lung was characterized by large sheets, multiple nodular high-density shadows, and 



Figure 1. CT scans of disease progression in a patient with primary mucinous adenocarcinoma of the lung. (A) Thoracic CT scan on April 30, 2012, revealing a space-occupying lesion of the right lung; (B) Thoracic CT scan following surgery in December 2012, revealing a metastasis mass in the left lung; (C) Thoracic CT scan following surgery on May 13, 2013, revealing that the left lung metastatic mass was enlarging. CT, computed tomography.

the limited patchy shadow was unchanged compared with that prior to treatment. Purified protein derivative, acid-fast stain test of sputum smear and T-SPOT.TB assays (Oxford Immunotec Ltd., Abingdon, UK; performed according to the manufacturer's protocol) were all negative. The respiratory symptoms became increasingly severe. Thoracic surgeons suggested a thoracic exploratory operation. The patient received a routine preoperative examination, and in May 2012, the patient underwent surgical treatment. The findings of the operation revealed that the whole right lung was distributed diffusely by a space-occupying lesion. Therefore, a right pneumonectomy was performed. Postoperative pathology demonstrated lung mucinous adenocarcinoma. The histopathology also revealed no mutations in epidermal growth factor receptor (EGFR). According to the 2009 tumor-node-metastasis classification by the IASLC (11-13), the pathological stage was determined to be cT4N0M0, IIIA (Fig. 2). The Eastern Cooperative Oncology Group
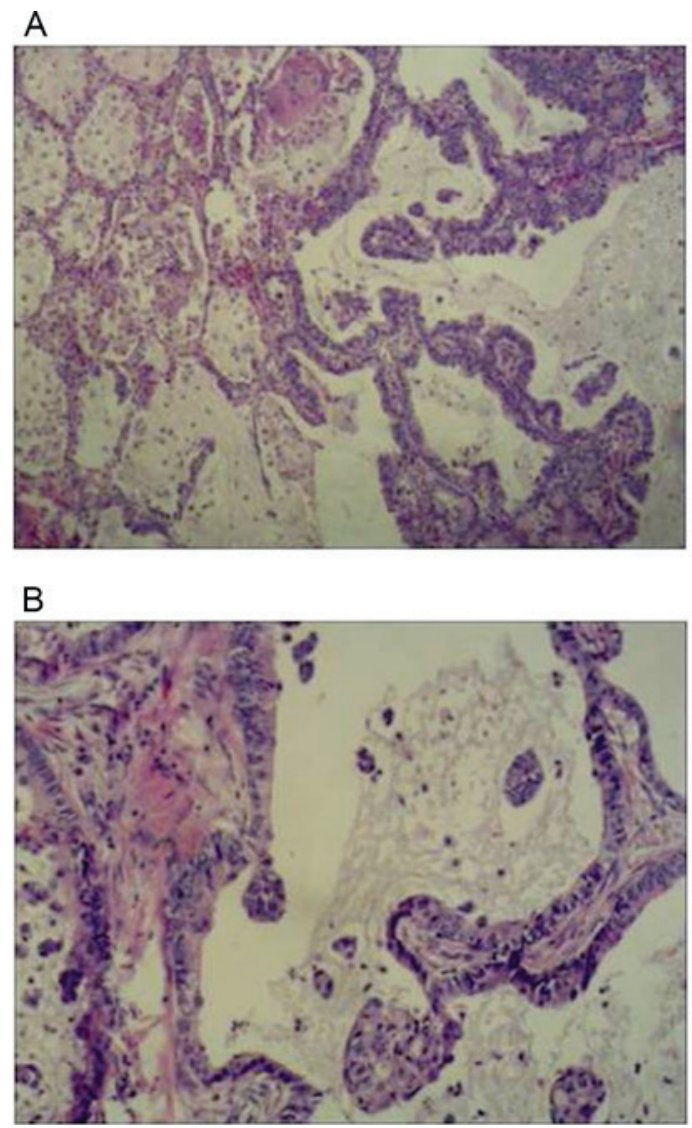

Figure 2. Hematoxylin and eosin staining of lung mucinous adenocarcinoma. (A) $\times 40$ objective, (B) x100 objective.

performance status was 1 . Adjuvant postoperative chemotherapy included four cycles of taxol $\left(135 \mathrm{mg} / \mathrm{m}^{2}\right.$ on day 1) and cisplatin (75 mg/m $\mathrm{m}^{2}$ on days $\left.1-4\right)$. The patient experienced grade II bone marrow suppression and grade III gastrointestinal reaction. The last administration of taxol and cisplatin chemotherapy was on September 7, 2012. In December 2012, chest CT re-examination revealed metastasis in the left lung (Fig. 1B). The patient received two cycles of chemotherapy based on pemetrexed and cisplatin as second-line treatment. The last administration of pemetrexed and cisplatin chemotherapy happened on January 12, 2013. Subsequently, the patient terminated chemotherapy and initiated treatment with Chinese herbs. On May 13, 2013, the patient received a chest CT re-examination (Fig. 1C). On August 27, 2013, a re-examining chest $\mathrm{CT}$ revealed metastasis in the left lung, which developed slowly (Fig. 3A and B). The symptom of a cough with white phlegm gradually appeared. The patient experienced tightness in the chest in March 2014, and re-examining chest CT revealed multiple metastases in the left lung (Fig. 3C and D). Therefore, the patient received treatment consisting of two cycles of chemotherapy with pemetrexed and cisplatin. The symptom of tightness in the chest disappeared following chemotherapy. Anti-tumor therapy was terminated again due to the financial situation of the patient's family. The patient was re-examined on June 12, 2014 (Fig. 3E and F), November 10, 2014 (Fig. 3G and H), February 6, 2015 (Fig. 4A), February 16, 2015 (Fig. 4B), March 5, 2015 (Fig. 4C and D) and March 25, 2015 (Fig. 4E and F). Metastasis outside the left lung did not 

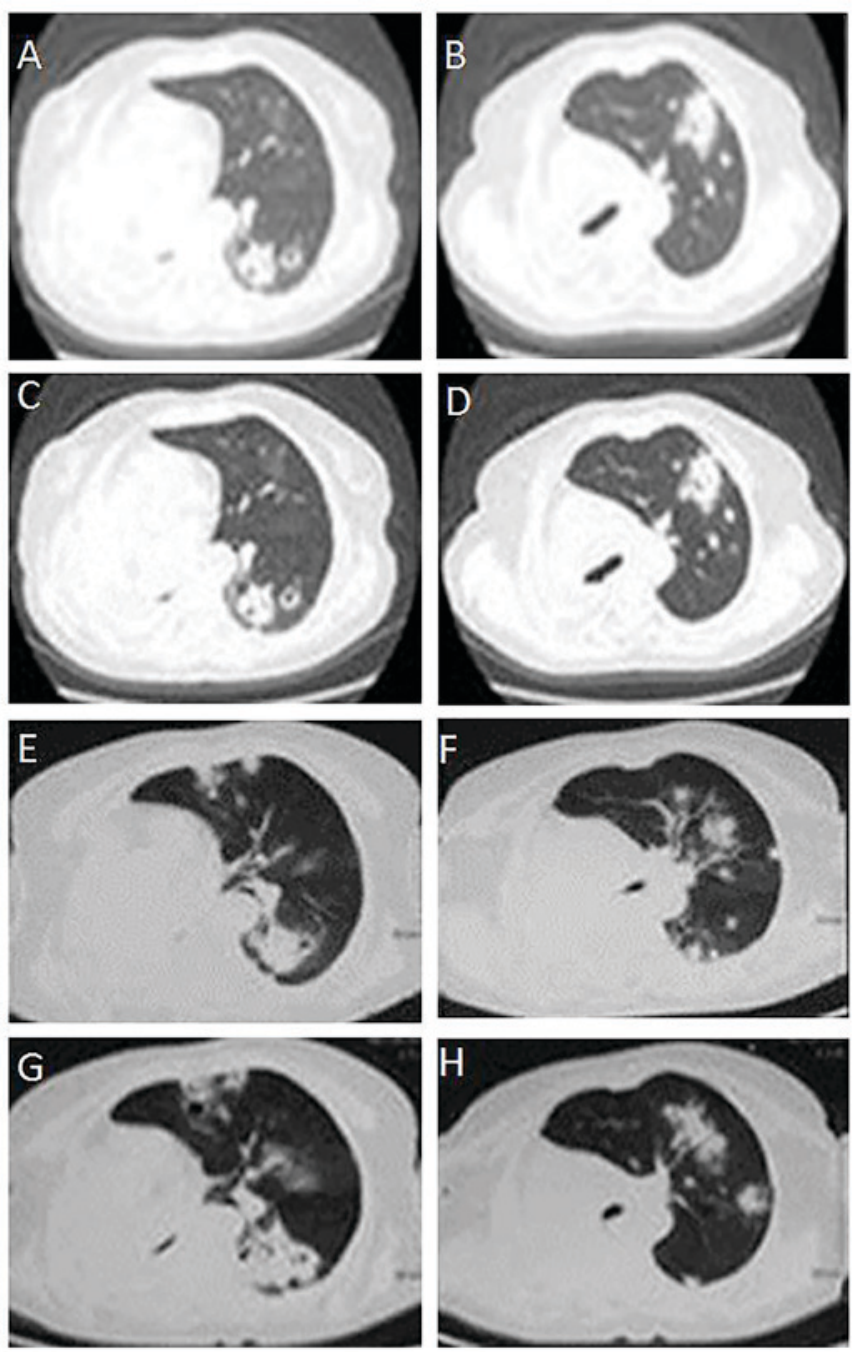

Figure 3. CT scans of the development of multiple metastases of the left lung in a patient with primary mucinous adenocarcinoma. (A and B) Thoracic CT scans following surgery in August 27,2013, revealing multiple metastases of the left lung. (C and D) Thoracic CT scans following surgery in March, 2014 revealing that the metastases of left lung were stable. (E and F) Thoracic CT scan following surgery in June 12, 2014, revealing an increase in the number of metastases of the left lung. ( $\mathrm{G}$ and $\mathrm{H}$ ) Thoracic $\mathrm{CT}$ scan following surgery on November 10, 2014, revealing further increases in the number of metastases of the left lung. CT, computer tomography.

appear. The patient succumbed to disease on May 18, 2015. Consent was obtained from the family of the patient.

\section{Discussion}

Mucinous adenocarcinoma is an uncommon histological subtype of primary lung adenocarcinoma. Mucus production is a typical feature of mucinous adenocarcinoma, which originates in stem cells with the potential of multidirectional differentiation and secrets mucus with different properties according to the differentiation microenvironment (6). Usually, the mucus secreted by cancer cells can be discharged as sputum, but if the growth of cancer cells is uncontrolled, it may cause excessive production of mucus, which obstructs the upper airways. In consequence, obstructive pneumonia may arise (14).

A retrospective review of the medical history of the patient of the present study revealed certain clinical features of lung

mucinous adenocarcinoma: i) During the course of the disease, the patient recurrently coughed with abundant white phlegm, despite the fact that the patient had no history of tobacco smoking or respiratory diseases, and sputum examination was negative. Thus, abundant white phlegm is associated with large quantities of mucus secreted by cancer cells. This feature reflects the consistency of the clinical symptoms with the biological behavior of cancer cells. ii) Pemetrexed was an effective second-line chemotherapy for the patient, since the symptom of chest tightness disappeared following two cycles of chemotherapy with pemetrexed and cisplatin, although cough and expectoration remained. Mucinous adenocarcinoma is a histological type of lung adenocarcinoma, and pemetrexed is used as first-line chemotherapy in patients with lung adenocarcinoma. Therefore, the variation of the clinical symptoms prior to and following treatment was associated with histopathology. iii) In the present patient, the lesions were confined to the lungs from the time of initial detection to mortality, and there was no metastasis to extrapulmonary organs. iv) The time span from diagnosis to mortality was $>3.5$ years, and the survival time was long despite the eight-cycle chemotherapy received by the patient. v) The rate of EGFR mutations is low in lung mucinous adenocarcinoma, as the patient had no mutation in EGFR from exons 18 to 21. vi) The clinical characteristics of the patient were cough, expectoration, fever, chest distress, no hemoptysis and pain.

The survival time of the patient was $>3.5$ years, as a result of a joint effort of the Departments of Oncology, Chest Surgery, 
Pathology and Imaging at Zhengzhou People's Hospital, which reflects the multidisciplinary team nature of oncotherapy.

In conclusion, lung mucinous adenocarcinoma is uncommon, but its prognosis is poor. Through the present case report, the clinical characteristics of lung mucinous adenocarcinoma were reviewed. Further understanding of this disease will help to improve the diagnosis and treatment. The identification of novel medicines and therapeutic methods for lung mucinous adenocarcinoma is urgent.

\section{References}

1. Ma NQ, Liu LL, Min J, Wang JW, Jiang WF, Liu Y, Feng YG, Su HC, Feng YM and Zhang HL: The effect of down regulation of calcineurin A $\alpha$ by lentiviral vector-mediated RNAi on the biological behavior of small-cell lung cancer and its bone metastasis. Clin Exp Metastasis 28: 765-778, 2011.

2. Liu Y, Zhang Y, Min J, Liu LL, Ma NQ, Feng YM, Liu D, Wang PZ, Huang DD, Zhuang Y and Zhang HL: Calcineurin promotes proliferation, migration, and invasion of small cell lung cancer. Tumour Biol 31: 199-207, 2010.

3. Travis WD, Brambilla E, Noguchi M, Nicholson AG, Geisinger KR, Yatabe Y, Beer DG, Powell CA, Riely GJ, Van Schil PE, et al: IInternational association for the study of lung cancer/american thoracic society/european respiratory society international multidisciplinary classification of lung adenocarcinoma. J Thorac Oncol 6: 244-285, 2011.

4. Mansuet-Lupo A, Bobbio A, Blons H, Becht E, Ouakrim H, Didelot A, Charpentier MC, Bain S, Marmey B, Bonjour P, et al: The new histologic classification of lung primary adenocarcinoma subtypes is a reliable prognostic marker and identifies tumors with different mutation status: The experience of a French cohort. Chest 146: 633-643, 2014.

5. Chen Z, Liu X, Zhao J, Yang $H$ and Teng X: Correlation of EGFR mutation and histological subtype according to the IASLC/ATS/ERS classification of lung adenocarcinoma. Int J Clin Exp Pathol 7: 8039-8045, 2014.
6. Marchetti A, Buttitta F, Pellegrini S, Chella A, Bertacca G, Filardo A, Tognoni V, Ferreli F, Signorini E, Angeletti CA and Bevilacqua G: Bronchioloalveolar lung carcinomas: K-ras mutations are constant events in the mucinous subtype. J Pathol 179: 254-259, 1996.

7. Travis WD, Brambilla E and Riely GJ: New pathologic classification of lung cancer: Relevance for clinical practice and clinical trials. J Clin Oncol 31: 992-1001, 2013.

8. Marchetti A, Buttitta F, Pellegrini S, Chella A, Bertacca G, Filardo A, Tognoni V, Ferreli F, Signorini E, Angeletti CA and Bevilacqua G: Bronchioloalveolar lung carcinomas: K-ras mutations are constant events in the mucinous subtype. J Pathol 179: 254-259, 1996

9. Nakaoku T, Tsuta K, Ichikawa H, Shiraishi K, Sakamoto H, Enari M, Furuta K, Shimada Y, Ogiwara H, Watanabe S, et al: Druggable oncogene fusions in invasive mucinous lung adenocarcinoma. Clin Cancer Res 20: 3087-3093, 2014.

10. Cai D, Li H, Wang R, Li Y, Pan Y, Hu H, Zhang Y, Gong R, Pan B, Sun Y and Chen H: Comparison of clinical features, molecular alterations, and prognosis in morphological subgroups of lung invasive mucinous adenocarcinoma. Onco Targets Ther 7: 2127-2132, 2014.

11. Goldstraw P: The 7th Edition of TNM in Lung Cancer: What now? J Thorac Oncol 4: 671-673, 2009.

12. Giroux DJ, Rami-Porta R, Chansky K, Crowley JJ, Groome PA, Postmus PE, Rusch V, Sculier JP, Shepherd FA, Sobin L, et al: The IASLC lung cancer staging project: Data elements for the prospective project. J Thorac Oncol 4: 679-683, 2009.

13. Rusch VW, Asamura H, Watanabe H, Giroux DJ, Rami-Porta R and Goldstraw P; Members of IASLC Staging Committee: The IASLC lung cancer staging project: a proposal for a new international lymph node map in the forthcoming seventh edition of the TNM classification for lung cancer. J Thorac Oncol 4: 568-577, 2009.

14. Higashiyama M, Doi O, Kodama K, Yokouchi H and Tateishi R: Cystic mucinous adenocarcinoma of the lung. Two cases of cystic variant of mucus-producing lung adenocarcinoma. Chest 101: 763-766, 1992. 